\title{
From pulpal stem cells to tooth repair: an emerging field for dental tissue engineering
}

\author{
Anne Baudry ${ }^{1,2}$, Emel Uzunoglu ${ }^{1,2}$, Benoit Schneider ${ }^{1,2}$, Odile Kellermann ${ }^{1,2}$ and Michel Goldberg ${ }^{1,2^{*}}$
}

\begin{abstract}
In current dental practices, traditional restorative approaches may have relatively limited long-term survival and may be associated to diverse complications, such as allergy, pulpitis, or periodontal pathologies. To overcome these shortcomings, novel innovative strategies have been envisioned for tooth repair. During the two last decades, the extensive advances in our understanding of tooth development as well as stem cell research provide the foundation for exciting opportunities in dental tissue engineering. The replacement of lost teeth by engineered dental tissue appears as a fascinating goal. However, the feasibility remains an intriguing question. Is the challenge to create a new tooth acting as a substitute for lost tooth or to regenerate only part of this organ that is enamel, dentin, or dental pulp? Is it possible to exploit stem cells for transplantation purposes to promote matrix formation and mineralization in the framework of endodontic treatment? Finally, investigating the functional properties of pulpal stem cells is however mandatory to envision novel therapeutic dental strategies. In this review, we summarize the current knowledge of stem cells used for dental tissue engineering and discuss the ensuing challenges for regenerative dentistry.
\end{abstract}

Keywords: Dental tissue engineering, Matrix mineralization, Stem cells

\section{Review \\ Introduction \\ Rationale for embryonic tooth-germ-based dental engineering}

During embryogenesis, reciprocal interactions between the oral epithelium and neural crest-derived ectomesenchyme lead to the initial formation of dental placode and afterward guide the building of a tooth through sequential morphogenetic events, i.e. bud, cap, bell (Cobourne et al. 2014). This tooth patterning involves a strictly spatio-temporal control of signaling pathways that sustain the interdependent inductive interactions, that is signal exchanges between epithelial and mesenchymal cells (Thesleff 2003). Epithelial cells are at the

\footnotetext{
* Correspondence: mgoldod@gmail.com

Anne Baudry and Emel Uzunoglu are co-first authors.

Odile Kellermann and Michel Goldberg are co-last authors.

${ }^{1}$ INSERM UMR-S 1124, Cellules souches, Signalisation et Prions, 75006 Paris,

France

${ }^{2}$ Faculté des Sciences Fondamentales et Biomédicales des Saints Pères,

Université Paris Descartes \& INSERM UMR-S 1124, Sorbonne Paris Cité, 45 rue des Saints Pères, Paris 75006, France
}

root of enamel-forming ameloblasts. Ectomesenchymal cells give rise to (i) odontoblasts, which synthesize the dentin extracellular matrix, and (ii) dental pulp cells assuming tooth homeostasis. During tooth organogenesis, odontogenic signals first emanate from the dental epithelium. Then, dental mesenchyme generates signals promoting the maturation of developing ameloblasts. Conceptually, tooth regeneration can thus be approached by taking into account these reciprocal epitheliomesenchymal interactions.

In 2004, murine tooth structures were successfully bioengineered either after implantation of dissociated rat tooth bud cell-seeded scaffolds in the rat omenta (ectopic site) (Dualibi et al. 2004) or after in vitro combination of non-dental mesenchymal cells with embryonic oral epithelium and transplantation of this primordium into mice adult jaw (Ohazama et al. 2004). Recombining the tissues lining the oral stomodeum and the mesenchyme seems to mimic developmental events and leads 
after implantation to the formation of tooth structures (enamel, dentin, pulp) with a morphology similar to that of natural teeth. Besides, tooth regeneration also implies restoration of the root with a periodontal ligament connected to the alveolar bone. Recently, mouse tooth germs reconstituted with the help of three-dimensional organ culture technologies (Ikeda et al. 2009; Nakao et al. 2007) or bioengineered tooth unit (Oshima et al. 2011) transplanted into the alveolar bone in the lost tooth region in an adult mouse yield tooth organ structures resembling those of native tooth. Because, tooth lost is the most common organ failure, these studies suggest that embryonic tooth germ used to generate bioengineered tooth holds promise for future tooth replacement. However, the inaccessibility of embryonic tooth germs and the need of autologous cells for tooth replacement limit such approach in routine clinical practices.

\section{Cell sources for tooth regeneration}

To overcome limitations associated with embryonic tooth germs, an alternative strategy relies on stem cells that could substitute for embryonic tooth germ in dental tissue engineering. In this context, a major challenge concerns the source, identification and isolation of stem cells potentially useful for transplantation or reconstitution approaches. In fact, stem cells are rare and are the unique cells endowed with the double capacities to self-renew and acquire specialized functions upon differentiation. These key properties render these cells tremendously attractive for repair and regeneration of deficient tissues and organs, including teeth.

Embryonic stem cells Pluripotent embryonic stem cells (ESCs), derived from the inner cell mass of mouse blastocysts, were shown to be capable in vitro to express ameloblast-specific proteins such as ameloblastin, amelogenin (Ning et al. 2010), or odontoblast-related mRNA like dentin sialophosphoprotein (DSPP) and dentin matrix acidic phosphoprotein-1 (DMP-1) (Li et al. 2007; Kawai et al. 2014) under suitable conditioned mediums. Furthermore, ESCs recombined with embryonic oral epithelium respond by expressing odontogenic genes (Ohazama et al. 2004). Despite the interesting potentialities of ESCs for dental tissue engineering, several major issues have to be dealt, in particular the ethical source of ESCs and their capacity to evolve into tumors (teratoma formation) when transplanted.

Mesenchymal stem cells Pluripotent stem cells are also present in the adult organism to maintain tissue homeostasis and to ensure tissue repair after lesion. These adult stem cells display a restricted lineage potential.
Interestingly, mesenchymal stem cells (MSCs) derived from mouse bone marrow have been shown to display an odontogenic potential (Ohazama et al. 2004; Li et al. 2007). Co-culture of these non-dental stem cells with oral epithelial cells derived from mouse embryos renders the MSCs able to express odontogenic genes such as Pax9, Msx1, Lhx7, DMP1, and DSPP. The transplantation of such cell mixture in murine renal capsule leads to the formation of several tooth-like structures. MSCs may thus represent an autologous source of cells for tooth regenerative research.

Dental stem cells Since 2000, several laboratories provided the evidence for the presence of stem cells in teeth. This includes dental pulp stem cells (DPSCs) that are heterogeneous cell populations derived from the pulp of the human permanent third molar (Gronthos et al. 2000). Other stem cells have been isolated from human exfoliated deciduous teeth (SHED) (Miura et al. 2003). SCAP cells are derived from the apical part of the papilla of growing tooth roots (Sonoyama et al. 2008). Our laboratory showed that the stem cells located in the open apical papilla of the rat molar have the capacity to divide after pulp exposure as shown by proliferating cell nuclear antigen immunostaining. These cells migrate from the central part of the pulp to the lateral subodontoblastic boundaries. Then, they slide from the root towards the coronal part of the pulp where they underwent terminal differentiation (Hirata et al. 2014), suggesting that apical progenitor cells are mobilized and contribute to pulp regeneration. In vitro, all these heterogeneous cell populations (DPSCs, SHEDs, SCAPs) have the ability to differentiate into odontoblasts, osteoblasts, and adipocytes as well as even into neuron-like cells. In vivo, they are able to form a dentin-like structure after transplantation with hydroxyapatite/tricalcium phosphate in immunocompromised mice (Huang et al. 2009). A few stem cells are also found in the dental follicle (DFSCs) (Morsczeck et al. 2005; Honda et al. 2010) and in the periodontal ligament of permanent tooth (PDLCs) (Seo et al. 2004) or deciduous tooth (DePDL) (Silvério et al. 2010). In vivo DFSC transplantation and co-implantation of SCAPs and PDLCs can lead to the formation of periodontal ligament-like tissues (Sonoyama et al. 2006; Yokoi et al. 2007). At the chair side, adult dental stem cells are easily accessible from extracted wisdom molars or their surrounding tissues. These dental stem cells may thus represent useful autologous cells for tooth engineering therapies.

Although potentially easily accessible, these dental stem cells are rare in teeth, as are others stem cells in adult tissue. It is now admitted that less than $1 \%$ of cells within a tissue are stem cells (Sloan and Waddington 2009). These stem cells are still difficult to detect and 
isolate. In vitro, they may also de-differentiate even after a limited number of passages. Before using these cells in routine practices of dentistry, further investigation for the identification, amplification, and maintenance of the lineage potential of such dental stem cells is required.

Induced pluripotent stem cells Facing the safety concerns of ESCs, and the difficulties to isolate and exploit adult stem cells for dental therapies in clinics, induced pluripotent stem cells (iPSCs) which could be another option to regenerate lost tooth or to repair a tooth injury. By over-expressing some transcription factors, including Oct3/4, Sox2, Nanog, Klf4, c-Myc, and Lin28, adult somatic cells may be reprogrammed into an ESC-like pluripotent state (Okita and Yamanaka 2010). In vivo, iPSCs were shown to further redifferentiate according to the site where the cells were implanted, indicating that cell environment plays a crucial role to orientate the cell fate. Many dental cells such as DPSCs, SHEDs, SCAPs, periodontal ligament, and gingival fibroblasts have been used to generate iPSCs (Hynes et al. 2015), and iPSCs derived from dental tissues thus represent a promising unlimited source of autologous cells for regenerative dentistry. However, the use of iPSCs in cell-based therapeutic approaches in dental clinical trials could be compromised because of the phenotypic instability of iPSC-derived differentiated cells (Goldberg 2011) and tumorigenesis effects (Takahashi and Yamanaka 2006).

\section{Stimulating natural dental repair based on the dual serotonergic/dopaminergic functions of pulpal stem cells}

Despite tremendous efforts to develop stem cell-based tooth regenerative strategies, the identification of intrinsic properties of pulpal stem cells is an unresolved question. Dental pulp stem cells are heterogeneous cell subpopulations likely composed of stem cells at different differentiation stages along odontoblastic lineage, which has broken their characterization. Another major drawback in the dental field is that the physiological signals governing the recruitment of pulpal stem cells for tooth repair upon injury still remain unknown.

A decade ago, our laboratory established clonal odontogenic cell lines with stem cell properties from first molar tooth germs of day 18 mouse embryos transgenic for an adenovirus-SV40 recombinant plasmid (pK4) (Priam et al. 2005). These dental pulp-derived cell lines are stable and maintain an undifferentiated phenotype under long-term standard culture conditions. Among the pulp-derived clones, the A4 cell line behaves as a multipotent mesoblastic stem cell. Upon in vitro appropriate induction, A4 cells can undergo unidirectional differentiation along either odonto/osteogenic, chondrogenic, or adipogenic lineage. Another clonal pulp cell line, H8, is a monopotent progenitor that displays a restricted potential of differentiation towards the odontoblastic fate (Lacerda-Pinheiro et al. 2012). In vivo, after implantation in a mouse incisor or rat molar, both $\mathrm{A} 4$ and $\mathrm{H} 8$ cells have the capacity to induce spatio-temporally controlled osteodentin formation and thus to promote efficient tooth repair (Lacerda-Pinheiro et al. 2012; Harichane et al. 2011). Thus, the isolation of A4 and $\mathrm{H} 8$ cell lines that both belong to the odontogenic lineage but differ in their differentiation potential demonstrates that multipotential and restricted lineage progenitors coexist in the dental pulp (Lacerda-Pinheiro et al. 2012). These two independent, stable, and homogenous pulp cell lines with precursor properties represent useful tools to search for the intrinsic properties of odontogenic stem cells.

By exploiting A4 and $\mathrm{H} 8$ cell lines, we disclosed for the first time the proper identity of odontogenic stem cells based on the expression of functional proteins. Both clones display a mixed serotonergic and dopaminergic phenotype (Baudry et al. 2015). This phenotype includes expression of all functional enzymes involved in serotonin (5-HT) and dopamine (DA) synthesis, catabolism, storage, and transport. Both cell lines also express the same definite repertoire of serotonergic and dopaminergic receptors of the $5-\mathrm{HT}_{2 \mathrm{~B}}, 5 \mathrm{HT}_{1 \mathrm{D}}$, and $5-\mathrm{HT}_{7}$ subtypes and dopaminergic $D_{1}$ and $D_{3}$ subtypes, respectively, which renders the cells competent to respond to circulating $5-\mathrm{HT}$ and DA. In vivo, we provided evidence that genuine pulpal stem cells with bioaminergic markers (double-positive staining for $5-\mathrm{HT}_{7} \mathrm{R}$ and the serotonin transporter SERT) exist within the pulp stroma of the rat molar but are scarce and are mobilized at the isthmus between the mesial and central parts of the pulp in response to injury.

We also uncovered that injury-activated platelets are the source of systemic 5-HT and DA necessary for the mobilization of genuine stem cells for dental repair (Baudry et al. 2015) (Fig. 1). After pulp injury, pulpal stem cellbased natural reparative dentin formation is impaired in fawn-hooded and reserpine-treated rats, two rat models whose blood platelets are deficient in bioamine storage. Finally, using selective antagonists of serotonergic or dopaminergic receptors, we demonstrated that $D_{1}$ and $D_{3}$ receptors as well as $5-\mathrm{HT}_{2 \mathrm{~B}}$ receptors and to a lesser extent $5-\mathrm{HT}_{7}$ receptors present on pulpal cells transduce platelet-derived 5-HT and DA signals and promote tooth repair. Thus, systemic 5-HT and DA from activated platelets represent "warning" signals necessary for tooth repair.

\section{Conclusions}

Promising researches on stem cells for dental tissue engineering were performed during the last 15 years, but 


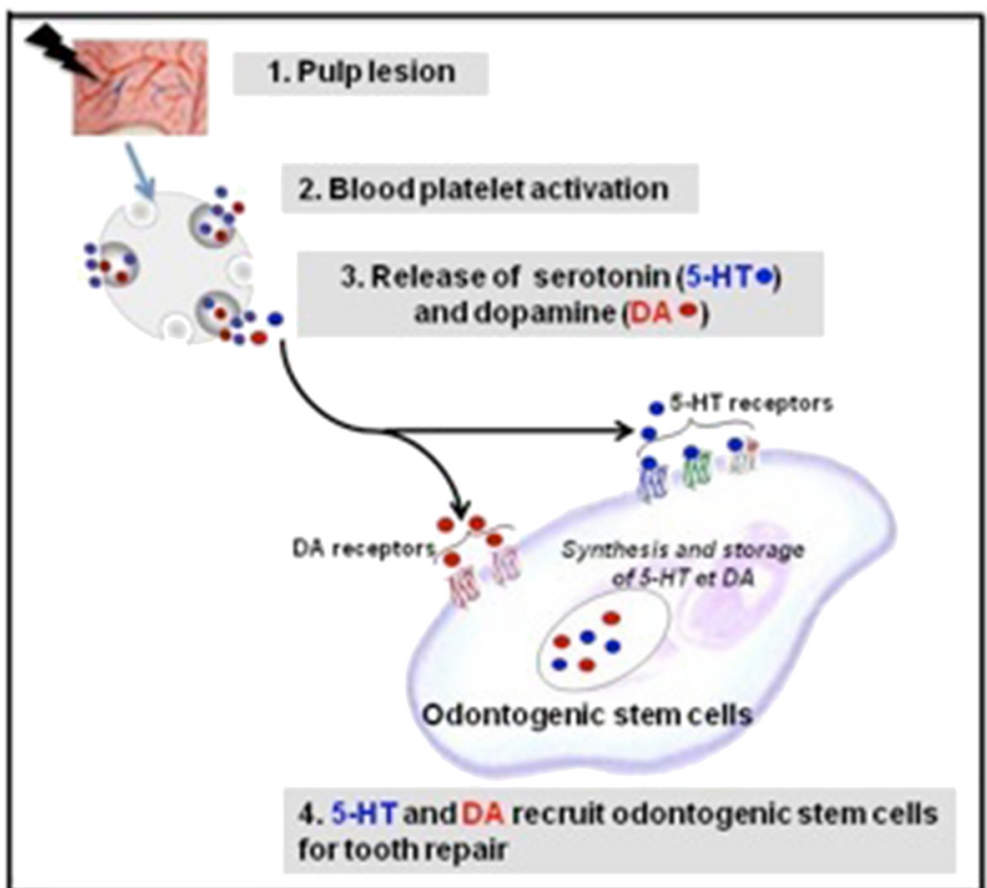

Fig. 1 Fundamental basis for novel approaches in dental repair

we are still far from the complex regeneration of a tooth. Several limitations persist and must be overcome before the use of stem cells in dental clinic, notably in terms of safety, capacity to isolate homogenous stem cell populations and to efficiently orientate their differentiation towards an odontogenic fate. Our recent work allowed to unveil the peculiar dual 5-HT/DA phenotype of mouse pulpal stem cells. The identification of specific functional proteins 5 -HT and DA receptors as well as the SERT present at their cell surface offers biomarkers to localize and isolate odontogenic stem cells from human dental pulp for future stem cell-based dental therapies. Finally, our demonstration of a link between the functional properties of odontogenic stem cell and their recruitment by platelet-derived 5-HT/DA signals for tooth repair paves the way to target resident stem cells and to envision novel therapeutic strategies in dentistry that is stimulating the natural reparative capacity of teeth.

\section{Competing interests}

The authors declare that they have no competing interests.

\section{Authors' contributions}

$A B$ is in charge of stem cell isolation and implantation and writing the review; EU is in charge of tooth implantation within the pulp and searching of databases; BS and OK are heads of the lab, in charge of language corrections and editing of the manuscript; MG is in charge of the concepts on tissue engineering. All the coauthors did substantial contributions to the conception, analysis of data, and interpretation. All authors read and approved the final manuscript.
Received: 29 December 2015 Accepted: 29 March 2016 Published online: 03 May 2016

\section{References}

Baudry A, Alleaume-Butaux A, Dimitrova-Nakov S, Goldberg M, Schneider B, Launay JM, et al. Essential roles of dopamine and serotonin in tooth repair: functional interplay between odontogenic stem cells and platelets. Stem Cells. 2015;33:2586-95.

Cobourne MT, Sharpe PT. Tooth development Chapter 3. In: AR, Hand and ME, Frank, editors. Fundamentals of Oral Histology and Physiology. Wiley Blackwell; 2014. p. 44-59.

Dualibi MT, Duailibi SE, Young CS, Bartlett JD, Vacanti JP, Yelick PC. Bioengineered teeth from cultured rat tooth bud cells. J Dent Res. 2004;83:523-8.

Goldberg M. Pulp healing and regeneration: more questions than answers. Adv Dent Res. 2011;23:270-4.

Gronthos S, Mankani M, Brahim J, Gehron Robey P, Shi S. Postnatal human dental pulp stem cells (DPSCs) in vitro and in vivo. Proc Natl Acad Sci U S A. 2000; 97:13625-30.

Harichane Y, Hirata A, Dimitrova-Nakov S, Granja I, Goldberg M, Kellermann O, et al. Pulpal progenitors and dentin repair. Adv Dent Res. 2011;23:307-12.

Hirata A, Dimitrova-Nakov S, Djole SX, Ardila H, Baudry A, Kellermann O, et al. Plithotaxis, a collective cell migration, regulates the sliding of proliferating pulp cells located in the apical niche. Connect Tissue Res. 2014;55 Suppl 1:68-72.

Honda MJ, Immaizumi M, Tsuchiya S, Morsczeck C. Dental follicle stem cells and tissue engineering. J Oral Sci. 2010;52:541-52.

Huang GTJ, Gronthos S, Shi S. Mesenchymal stem cells derived from dental tissues vs. Those from other sources. J Dent Res. 2009;88:792-806.

Hynes K, Menichanin D, Bright R, Ivanovski S, Hutmacher DW, Gronthos S, et al. Induced pluripotent stem cells: a new frontier for stem cells in dentistry. J Dent Res. 2015;94:1508-15.

Ikeda E, Morita R, Nakao K, Ishida K, Nakamura T, Takano-Yamamoto T, et al. Fully functional bioengineered tooth replacement as an organ replacement therapy. Proc Natl Acad Sci U S A. 2009;106:13475-80.

Kawai R, Ozeki N, Yamaguchi H, Tanaka T, Nakata K, Mogi M, et al. Mouse ES cells have a potential to differentiate into odontoblast-like cells using hanging drop method. Oral Dis. 2014;20:395-403. 
Lacerda-Pinheiro S, Dimitrova-Nakov S, Harichane Y, Souyri M, Petit-Cocault L, Legrès $L$, et al. Concomitant multipotent and unipotent dental pulp progenitors and their respective contribution to mineralised tissue formation. Eur Cell Mater. 2012;23:371-86.

Li ZY, Chen L, Liu L, Lin YF, Li SW, Tian WD. Odontogenic potential of bone marrow mesenchymal stem cells. J Oral Maxillofac Surg. 2007;65:494-500.

Miura M, Gronthos S, Zhao M, Lu B, Fisher LW, Gehron Robey P, et al. SHED: Stem cells from human exfoliated deciduous teeth. Proc Natl Acad Sci U S A. 2003;1000:5807-12.

Morsczeck C, Gotz W, Schierholz J, Zeilhofer F, Kuhn U, Mohl C, et al. Isolation of precursor cells (PCs) from human dental follicle of wisdom teeth. Matrix Biol. 2005;24:155-65.

Nakao K, Morita R, Saji Y, Ishida K, Tomita Y, Ogawa M, et al. The development of a bioengineered organ germ method. Nat Methods. 2007;4:227-30.

Ning F, Guo Y, Tang J, Zhou J, Zhang H, Lu W, et al. Differentiation of mouse embryonic stem cells into dental epithelial-like cells induced by ameloblasts serum-free conditioned medium. Biochem Biophys Res Commun. 2010;394:342-7.

Ohazama A, Modino SAC, Miletich I, Sharpe PT. Stem-cell-based tissue engineering of murine teeth. J Dent Res. 2004;83:518-22.

Okita K, Yamanaka S. Induction of pluripotency by defined factors. Exp Cell Res. 2010;316:2565-70.

Oshima M, Mizuno M, Imamura A, Ogawa M, Yasukawa M, Yamazaki H, et al. Functional tooth regeneration using a bioengineered tooth unit as a mature organ replacement regenerative therapy. PLoS One. 2011;6:e21531.

Priam F, Ronco V, Locker M, Bourd K, Bonnefoix M, Duchêne T, et al. New cellular models for tracking the odontoblast phenotype. Arch Oral Biol. 2005:50:271-7.

Seo BM, Miura M, Gronthos S, Barthold PM, Batouli S, Brahim J, et al. Investigation of multipotent postnatal stem cells from human periodontal ligament. Lancet. 2004;364:149-55.

Silvério KG, Rodrigues TL, Coletta RD, Benevides L, Da Silva JS, Casati MZ, et al. Mesenchymal stem cell properties of periodontal ligament cells from deciduous and permanent teeth. J Periodontol. 2010;81:1207-15.

Sloan AJ, Waddington RJ. Dental pulp stem cells: what, where, how? Int J Paediat Dent. 2009;19:61-70.

Sonoyama W, Liu Y, Fang D, Yamaza T, Seo BM, Zhang C, et al. Mesenchymal stem cell mediated functional tooth regeneration in swine. PLoS One. 2006;: : 79 .

Sonoyama W, Liu Y, Yamaza T, Tuan RS, Wang S, Shi S, et al. Characterization of apical papilla and its residing stem cells from human immature permanent teeth-a pilot study. J Endod. 2008;34:166-71.

Takahashi K, Yamanaka S. Induction of pluripotent stem cells from mouse embryonic and adult fibroblast cultures by defined factors. Cell. 2006; 126:663-76.

Thesleff I. Epithelial-mesenchymal signalling regulating tooth morphogenesis. J Cell Sci. 2003;116:1647-8.

Yokoi T, Saito M, Kiyono T, Iseki S, Kosaka K, Nishida E, et al. Establishment of immortalized dental follicle cells for generating periodontal ligament in vivo. Cell Tissue Res. 2007;327:301-11.

\section{Submit your manuscript to a SpringerOpen ${ }^{\circ}$ journal and benefit from:}

- Convenient online submission

- Rigorous peer review

- Immediate publication on acceptance

- Open access: articles freely available online

- High visibility within the field

- Retaining the copyright to your article 